\title{
Ultra slow muon microscopy by laser resonant ionization at J-PARC, MUSE
}

\author{
Y. Miyake • Y. Ikedo • K. Shimomura • P. Strasser • N. Kawamura • K. Nishiyama • \\ A. Koda • H. Fujimori • S. Makimura • J. Nakamura • T. Nagatomo • R. Kadono • \\ E. Torikai • M. Iwasaki • S. Wada • N. Saito • K. Okamura · K. Yokoyama • T. Ito • \\ W. Higemoto
}

Published online: 29 January 2013

(C) The Author(s) 2013. This article is published with open access at Springerlink.com

\begin{abstract}
As one of the principal muon beam line at the J-PARC muon facility (MUSE), we are now constructing a Muon beam line (U-Line), which consists of a large acceptance solenoid made of mineral insulation cables (MIC), a superconducting curved transport solenoid and superconducting axial focusing magnets. There, we can extract $2 \times 10^{8} / \mathrm{s}$ surface muons towards a hot tungsten target. At the U-Line, we are now establishing a new type of muon microscopy; a new technique with use of the intense ultra-slow muon source generated by resonant ionization of thermal
\end{abstract}

Proceedings of the 6th International Conference on Laser Probing (LAP 2012), Paris, France, 4-8 June 2012.

Y. Miyake $(\varangle) \cdot$ Y. Ikedo · K. Shimomura · P. Strasser · N. Kawamura · K. Nishiyama · A. Koda · H. Fujimori · S. Makimura · J. Nakamura · T. Nagatomo · R. Kadono Muon Science Laboratory, High Energy Accelerator Research Organization (KEK), 1-1 Oho, Tsukuba Ibaraki, 305-0801, Japan e-mail: yasuhiro.miyake@kek.jp

Y. Miyake · Y. Ikedo · K. Shimomura · P. Strasser · N. Kawamura · K. Nishiyama · A. Koda · H. Fujimori - S. Makimura - J. Nakamura - T. Nagatomo · R. Kadono · T. Ito · W. Higemoto Muon Section, Materials and Life Science Division, J-PARC Center, 2-4 Shirane Shirakata, Tokai-mura, Naka-gun, Ibaraki 319-1195, Japan

E. Torikai

Faculty of Engineering, Yamanashi University, Kofu, Yamanashi, 4008511, Japan

M. Iwasaki · K. Yokoyama

Advanced Meson Science Laboratory, RIKEN Nishina Center, Wako, Saitama 351-0198, Japan

S. Wada $\cdot$ N. Saito

Advanced Science Institute, RIKEN, 2-1 Hirosawa, Wako, Saitama, 351-0198, Japan

K. Okamura

Megaopto Co., Ltd., RIKEN-WAKO Incubation Plaza 301, Wako, Saitama 351-0104, Japan

T. Ito $\cdot$ W. Higemoto

Advanced Science Research Center, Japan Atomic Energy Agency,

Tokai, Ibaraki 319-1195, Japan 
Muonium (designated as $\mathrm{Mu}$; consisting of a $\mu^{+}$and an $\mathrm{e}^{-}$) atoms generated from the surface of the tungsten target. In this contribution, the latest status of the Ultra Slow Muon Microscopy project, fully funded, is reported.

Keywords J-PARC • Muon • Ultra slow muon • VUV • Pulsed laser • Resonant ionization

\section{Introduction}

The surface muon beam $\left(\mu^{+}, 30 \mathrm{MeV} / \mathrm{c}\right)$ which has been used for the studies of condensed matter physics or chemistry is conventionally obtained from the decay of positive pions $\left(\pi^{+}\right)$stopped near the surface of the pion production target in the proton beam line and has large energy broadening with an implantation depth of 0.1 to $1 \mathrm{~mm}$. Despite the name of surface muon, it has been used as a probe for bulk phenomena rather than surface phenomena. In these two decades, a new method to generate ultra-slow muon beam with energy $0.2 \mathrm{eV}$ has been developed at KEK and RIKEN-RAL, utilizing the resonant ionization technique [1]. However, its yield of 20/s maximally obtained [2] was not even enough for the researches in material science. In order to obtain a sufficient yield for the variety of surface, and nano-science, it was essential to embark on a new project at J-PARC MUSE (Muon Science Establishment), where the world strongest pulse muon source is available.

\section{D-Line, the strongest pulsed muon beamline}

For Phase 1 at J-PARC MUSE, we managed to install a superconducting decay/surface beam line, so called, D-Line, with a modest-acceptance pion injector of about $45 \mathrm{mSr}$, where either surface muons, or decay muons $\left(5-120 \mathrm{MeV} / \mathrm{c}, \mu^{+}\right.$ and $\mu^{-}$) that are obtained through the in-flight decay of $\pi^{+} / \pi^{-}$confined by a strong longitudinal magnetic field of several tesla from a superconducting decay solenoid magnet, can be extracted. Since the first delivery of the surface muon beam to the experimental area on September 26th, 2008, the muon beam commissioning had been continued. In November 2009, the surface muon extraction rate was significantly increased up to $1.8 \times 10^{6} / \mathrm{s}$ with a $120 \mathrm{~kW}$ proton beam from the $3 \mathrm{GeV}$ synchrotron (RCS). The achieved intensity was equivalent or even larger than that $\left(1.2 \times 10^{6} / \mathrm{s}\right)$ at the RIKEN-RAL muon facility [3]. This is the reason why the world strongest pulsed muon source was claimed to be achieved at MUSE even by using only a $120 \mathrm{~kW}$ proton beam. This intensity would correspond to $1.5 \times 10^{7} / \mathrm{s}$ surface muons when in the future the proton beam power will reach $1 \mathrm{MW}$ [4]. Since November 26th, 2010, the proton beam power from the RCS has steadily increased up to $220 \mathrm{~kW}$, consequently delivering $3.2 \times 10^{6} / \mathrm{s}$ of intense surface muon or several $10^{6} / \mathrm{s}$ of decay muon beam, until the earthquake on March 11th, 2011.

\section{U-Line, dedicated for the ultra slow muon}

In addition to D-Line, we were funded to install the second muon beamline, so called U-Line, which consists of a large acceptance solenoid made of mineral 


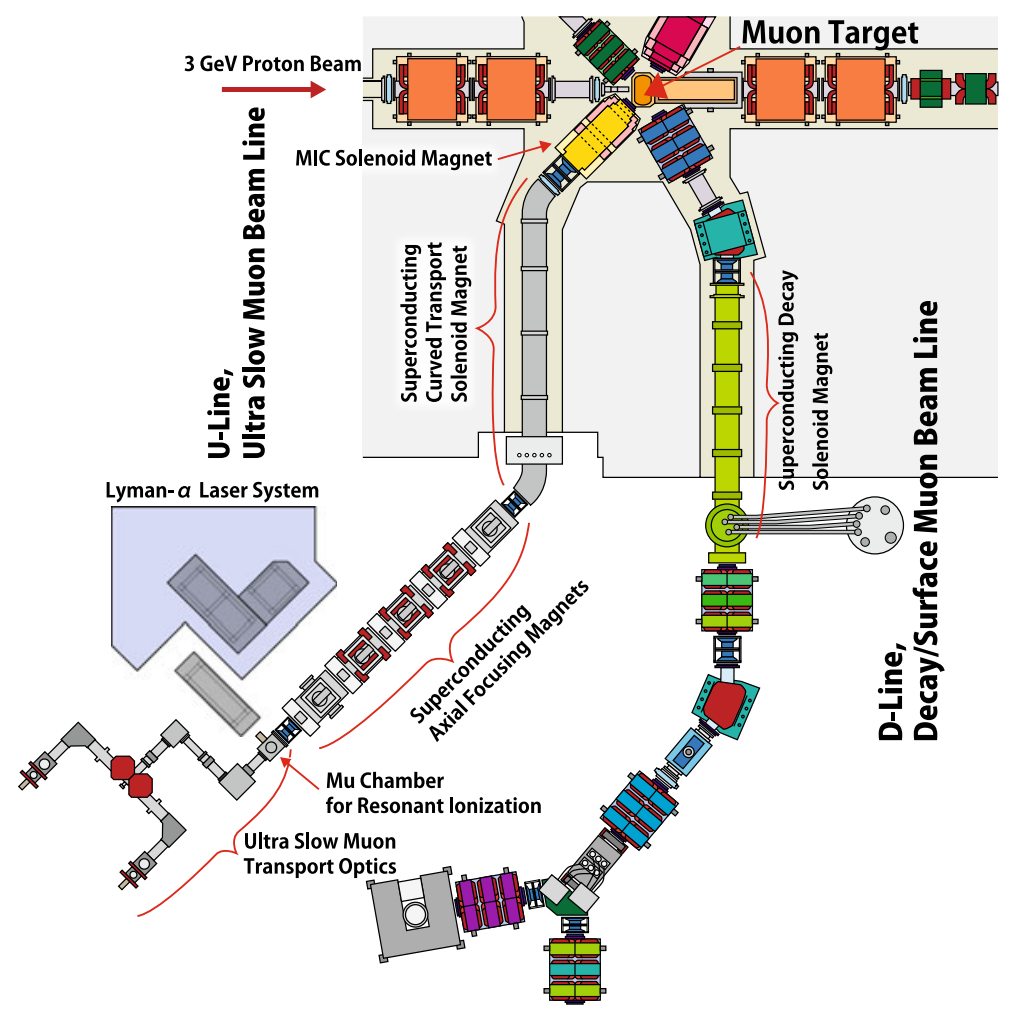

Fig. 1 A layout of J-PARC MUSE, D-Line and U-Line in the Materials and Life science experimental Facility (MLF) for neutron and muon

insulation cables (MIC), a superconducting curved transport solenoid magnet and a superconducting axial focusing magnets system. There, we can collect surface muons with a large acceptance of $400 \mathrm{mSr}$. Compared to the conventional beamlines such as D-Line, the large acceptance of the front-end solenoid will allow for the capture of more than 10 times intensity pulsed muons [5]. With a muon capture of $5 \times 10^{8} / \mathrm{s}$ surface muons, we can collect $2 \times 10^{8} / \mathrm{s}$ surface muons on the $\mathrm{W}$ target in the $\mathrm{Mu}$ chamber, with an approximate transport efficiency of $40 \%$. The U-Line components of the superconducting curved and axial focusing magnets were already fabricated and those commissioning will be performed in the autumn of 2012 (Fig. 1).

\section{Ultra slow muon microscopy}

Ultra slow muons are generated by resonant ionization of thermal $\mathrm{Mu}$ atoms generated from the surface of a hot tungsten foil, placed at the intense surface muon beam line. In order to efficiently ionize the $\mathrm{Mu}$ near the $\mathrm{W}$ surface, we adopted a resonant ionization scheme via the $\{1 \mathrm{~S}-2 \mathrm{P}$-unbound $\}$ transition. In order to induce $1 \mathrm{~s}-2 \mathrm{p}$ transitions, Lyman- $\alpha$ light of $122.088 \mathrm{~nm}$ is needed. To generate such Lyman- $\alpha$ (VUV;Vacuum Ultra Violet) laser, we have been adopting the resonant four-wave 


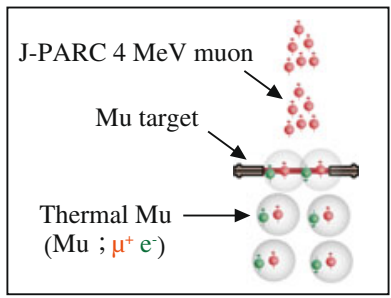

Mu generator

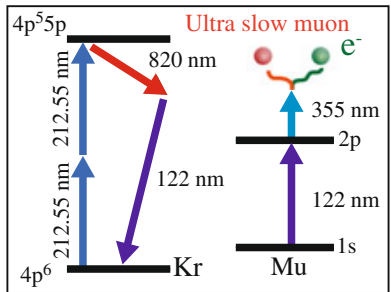

Lyman- $\alpha$ laser generation and Mu dissociation by laser resonant ionization method

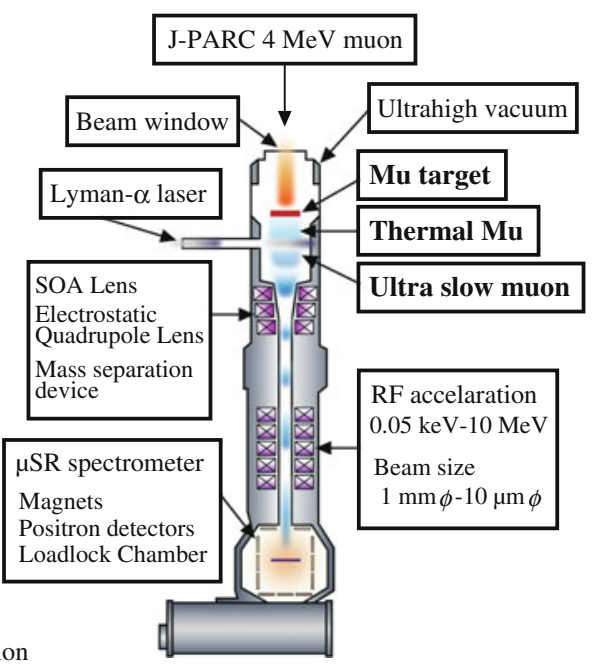

Fig. 2 A concept of the ultra low muon microscopy

frequency mixing $\left(\omega_{V U V}=2 \omega_{r}-\omega_{t}\right)$ where two $212.5 \mathrm{~nm}$ photons $\left(\omega_{r}\right)$ are used for two-photon resonant excitation of the $4 \mathrm{P}^{5} 5 \mathrm{P}[5 / 2]$ state in $\mathrm{Kr}$, subtracted by a photon with a tunable difference wavelength $\left(\omega_{t} ; 820 \mathrm{~nm}\right)$, rather than the frequency tripling (non-resonant) of $366 \mathrm{~nm}$ photons, of which conversion efficiency is in the order of $10^{-5-6}$. In our new laser system developed by Wada et al. [6], $\omega_{r}(100 \mathrm{~mJ} / \mathrm{p}, 2 \mathrm{~ns})$ is generated as the 5 th harmonics of $1062 \mathrm{~nm}(1 \mathrm{~J} / \mathrm{p})$, which consists of all solid state laser system, such as diode laser, fiber amplifier, regeneration amplifier, OPA, OPG etc., generating expectedly more than $71 \mu \mathrm{J} / \mathrm{cm}^{2}$ Lyman- $\alpha$ light of the saturation intensity for the Doppler broadened $\mathrm{Mu}$ at $2000 \mathrm{~K}$.

Comparing the surface muon intensity between RIKEN-RAL $\left(1.2 \times 10^{6} / \mathrm{s}\right)$ and ULine $\left(2 \times 10^{8} / \mathrm{s}\right)$, we can gain a factor of 160 . Taking into account the repetition rate of the pulsed laser system and the muon beam, we can also gain a factor of two, since both the laser and the muon beam can be synchronized to $25 \mathrm{~Hz}$ at MUSE, whereas the muon beam $(50 \mathrm{~Hz})$ is synchronized to every second laser pulse at the RIKENRAL facility. Moreover, the new solid state laser system is designed to generate an intense Lyman- $\alpha$ light of more than $100 \mu \mathrm{J} / \mathrm{cm}^{2}$ needed to saturate the transition of $\mathrm{Mu}$ from the $1 \mathrm{~S}$ state to the $2 \mathrm{P}$ state [6], whereas only about $1 \mu \mathrm{J} / \mathrm{cm}^{2}$ of the Lyman- $\alpha$ light was produced at RIKEN-RAL. Consequently, we may expect, as a maximum, $0.6 \times 10^{6} / \mathrm{s}$ of the ultra-slow $\mu^{+} / \mathrm{s}$ at MUSE (Fig. 2). This work was supported by a Grant-in-Aid for Scientific Research on Innovative Areas "Ultra Slow Muon" (No. 23108002) of the Ministry of Education, Culture, Sports, Science, and Technology, Japan and its commissioning will be performed in the winter of 2012.

\section{Summary}

At J-PARC MUSE, we are installing the U-Line which consists of a large acceptance solenoid made of mineral insulation cables (MIC) and a superconducting curved 
transport solenoid and 6 sets of superconducting axial focusing magnets. There, we can collect surface muons with a large acceptance of $400 \mathrm{mSr}$. Finally, we are expecting a rate of $0.6 \times 10^{6}$ ultra slow muons/s out of the $2 \times 10^{8}$ surface muons/s. When the production of intense ultra-slow muon source is realized, the use of its short-range penetration depth (eg. $1 \mathrm{~nm}$ resolution at a penetration of $1 \mathrm{~nm}$, and $10 \mathrm{~nm}$ at a penetration of $6 \mathrm{~nm}$ in Gold) will allow muon science to be expanded towards a variety of new scientific fields such as,

1) Surface/boundary magnetism utilizing its spin polarization and unique timewindow.

2) Surface chemistry, utilizing a feature of a light isotope of hydrogen; such as catalysis reactions.

3) Precise atomic physics such as QED,

4) Ion sources towards $\mu^{+} \mu^{-}$collider experiments in high-energy physics.

Open Access This article is distributed under the terms of the Creative Commons Attribution License which permits any use, distribution, and reproduction in any medium, provided the original author(s) and the source are credited.

\section{References}

1. Ghandi, K., Miyake, Y.: Modern slow muon beam production techniques. In: Hatano, Y., Katsumura, Y., Mozumder, A. (eds.) Charged Particle and Photon Interactions with MatterRecent Advances, Applications, and Interfaces, pp. 169-208. CRC Press, Taylor \& Francis, Boca Raton (2010)

2. Bakule, P., Matsuda, Y., Miyake, Y., Nagamine, K., Iwasaki, M., Ikedo, Y., Shimomura, K., Strasser, P., Makimura, S.: Nucl. Instrum. Methods Phys. Res., B Beam Interact. Mater. Atoms 266, 335-346 (2008)

3. Nagamine, T., Matsuzaki, K., Ishida, I., Watanabe, S.N., Nakamura, R., Kadono, N., Kawamura, S., Sakamoto, M., Iwasaki, M., Tanase, M., Kato, K., Kurosawa, G.H., Eaton, H.J., Jones, G., Thomas, G., Williams, W.G.: Hyperfine Interact. 101/102, 521 (1996)

4. Miyake, Y., Shimomura, K., Kawamura, N., Strasser, P., Makimura, S., Koda, A., Fujimori, H., Nakahara, K., Kadono, R., Kato, M., Takeshita, S., Nishiyama, K., Higemoto, W., Ishida, K., Matsuzaki, T., Matsuda, Y., Nagamine, K.: Physica B 404, 957-961 (2009)

5. Nakahara, K., Miyake, Y., Shimomura, K., Strasser, P., Nishiyama, K., Kawamura, N., Fujimori, H., Makimura, S., Koda, A., Nagamine, K., Ogitsu, T., Yamamoto, A., Adachi, T., Sasaki, K., Tanaka, K., Kimura, N., Makida, Y., Ajima, Y., Ishida, K., Matsuda, Y.: AIP Conf. Proc. 981, 312-314 (2007)

6. Saito, N., Wada, S., Okamura, K., Iwasaki, M.: In: Reports on the 427th Topical Meeting of the Laser Society of Japan Development of Short-Wavelength Radiations and Their Applications, No. RTM-12-15, 49-54 July (2012) 